\title{
Electrically Reconfigurable
} Microwave Metasurfaces With Active Lumped Elements: A Mini Review

\author{
Linda Shao and Weiren Zhu* \\ Department of Electronic Engineering, Shanghai Jiao Tong University, Shanghai, China
}

Metasurfaces, a kind of two-dimensional artificially engineered surfaces consist of subwavelength unit cells, have recently attracted tremendous attention, owing to their exotic abilities for tailoring electromagnetic responses. With active lump elements incorporated into the design of metasurfaces, dynamic reconfigurabilities enabled by external stimuli could be realized, offering opportunities for the dynamic manipulation of electromagnetic waves. In this mini review, we present a brief review on the recent progress of electrically reconfigurable metasurfaces at microwave frequencies. A brief discussion will also be given with our outlook on future development direction and possible challenges in this interesting field.

OPEN ACCESS

Edited by: Xiao Lin,

Zhejiang University, China

Reviewed by:

Shulin Sun,

Fudan University, China

Cai Tong,

Zhejiang University, China

*Correspondence:

Weiren Zhu

weiren.zhu@sjtu.edu.cn

Specialty section:

This article was submitted to Metamaterials,

a section of the journal

Frontiers in Materials

Received: 01 April 2021 Accepted: 28 May 2021

Published: 11 June 2021

Citation:

Shao L and Zhu W (2021) Electrically

Reconfigurable Microwave

Metasurfaces With Active Lumped

Elements: A Mini Review.

Front. Mater. 8:689665.

doi: 10.3389/fmats.2021.689665
Keywords: Metasurface, Meta-devices, PIN diodes, Reconfigurable metasurface, Varactors

\section{INTRODUCTION}

Metamaterials (Liu and Zhang, 2011; Zheludev and Kivshar, 2012), a kind of artificial electromagnetic media constructed with sub-wavelength units, have attended considerably increasing interests in both scientific and engineering communities. Metasurfaces ( $\mathrm{Yu}$ et al., 2011; Sun et al., 2012; Chen et al., 2016; Sun et al., 2019; Akram et al., 2020) are the twodimensional equivalents of metamaterials, formed by periodic or non-periodic arrangement of subwavelength elements in an ultrathin film, which possess flexible capability to control the amplitude, phase, and polarization state of electromagnetic(EM) waves. Versatile functionalities, such as perfect absorption, anomalous reflection, and focusing, can be realized with the help of metasurfaces (Akram et al., 2019; Shao et al., 2019). While metasurfaces could be artificially designed with various functionalities, the particular functionality is typically fixed after the design of the metasurface (Li Z. et al., 2020). The functionalities of metasurfaces are able be adjusted by loading active elements into the designs (Cui et al., 2014; Luo et al., 2016; Zhang et al., 2020a). On account of the possibilities to independently control each meta-atom by the embedded active lumped element, these meta-atoms can be assembled to form electrically reconfigurable metasurfaces with tunable amplitude/phase profiles. For example, it is suitable for reconfigurable metasurfaces to be constructed with meta-atoms incorporated with voltage-driven elements (such as PIN diodes (Ghosh and Srivastava, 2016), varactors (Wang et al., 2019), graphene (Zhang et al., 2020b)), so that electromagnetic characteristics can be dramatically tuned through varying applied voltages. By accurately determining the voltages applied on different meta-atoms, these meta-devices can realize desired functions for dynamical wave manipulation. In this mini review, we briefly review the major achievements in electrically reconfigurable microwave metasurfaces with different loaded active lump elements. We will also give a brief discussion with our perspectives on future developments and challenges in this area. 


\section{METASURFACES WITH PIN DIODES}

\subsection{Uniformly Tuning With PIN Diodes}

The tunablity of microwave metasurfaces can be enabled by incorporating PIN diodes in the structure design. A tunable circuit analog absorber whose equivalent thickness is electronically changed by employing PIN diodes to control the RF signal path, which results in absorbing frequency switching between $0.85-1.88$ and 2.66-5.23 GHz (Qi et al., 2012). Xu et al. demonstrated a tunable metamaterial absorber, whose reflection responses can be adjusted over $2-18 \mathrm{GHz}$ frequency range by changing the bias voltage on the PIN diode array (Xu et al., 2012). Recently, switchable perfect absorber/reflectors with a PIN diode integrated active frequency selective surface have attracted enormous interest (Yoo and Lim, 2014; Ghosh and Srivastava, 2016). In 2019, Zhao et al. proposed an electrically reconfigurable metasurface that can be switched between reflection and absorption modes by loading PIN diodes (Zhao et al., 2019). Perfect absorption occurs when the impedance of the dielectric substrate matches well with the free-space impedance while total reflection occurs when they mismatch. Similarly, switchable transmissive/absorptive metasurfaces have also been reported
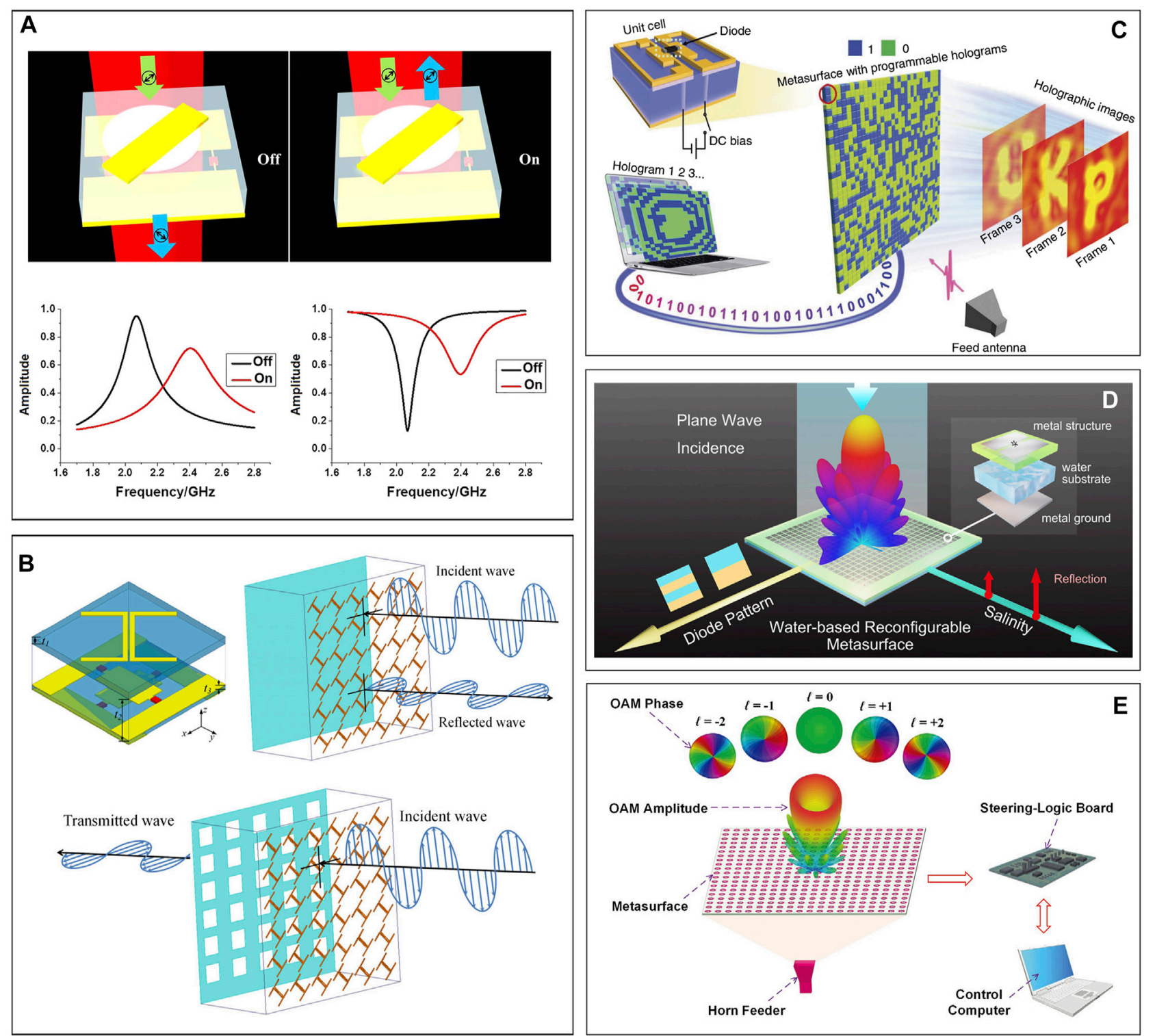

FIGURE 1 | (A) Active metasurface for reconfigurable transmissions/reflections and cross polarization conversions. Reproduced from Tao et al. (2017) with permission from AIP Publishing. (B) Reconfigurable metasurface for polarization and propagation Manipulation. Reproduced from Li Y. et al. (2019) with permission from IEEE. (C) Reprogrammable coding metasurface holograms. Reproduced from Li et al. (2017) with permission from Spring Nature. (D) Reconfigurable water-based metasurface integrated with PIN diodes. Reproduced from Chen et al. (2019a) with permission from AIP Publishing. (E) Transmissive programmable metasurface for multimode OAM generation. Reproduced from Bai et al. (2020) with permission from John Wiley and Sons. 
(Li Y. et al., 2020). Li et al. designed a tunable metadevice based on coupled-mode theory, which can be switched from perfect transparency to perfect absorption under the control of an external voltage applied to the PIN diode. In addition, flexible structures of unit cell offer another straightforward way to realize reconfigurable metadevices. Metasurfaces have shown powerful abilities in controlling the reflections, transmissions, and polarizations of EM waves, independently. A reconfigurable polarization converter is proposed, which based on an activeelement-controlled metasurface without extra bias network ( $\mathrm{Li}$ et al., 2016). It achieves circular-linear polarization conversion when the PIN diodes are switched to OFF. Flexible structures of unit cell offer another straightforward way to realize reconfigurable metadevices. Metasurfaces have shown great abilities in controlling the reflections, transmissions, and polarizations of EM waves, independently. As shown in Figure 1A, Tao et al. (2017) proposed a single active metasurface which is exploited to reconfigure propagating states of EM waves. By switching the status of PIN diodes embeded in each unit cell, the metadevice can not only switch its EM properties between the reflection and transmission mode, but also realize cross linearized polarization conversion. Very recently, Li Y. et al. (2019) demonstrated a multi-functional reconfigurable metasurface, which can control both the propagation and the polarization of EM wave. By controlling the bias voltage, it can switch its function among the reflectiontype converter, the transmission-type converter and the transparent structure (Figure 1B).

\subsection{Non-uniformly Tuning With PIN Diodes}

In order to achieve more elaborate functionalities, the locally applied uniformly tuning voltages are allowed to be different for each unit cell in the electrically reconfigurable metasurface. It enables more tunable applications to be achieved, such as multibeam generation (Huang et al., 2017a), tunable reflection (Wan et al., 2016; Yang et al., 2016), beam diffusion (Yang et al., 2016; Huang et al., 2017a), beam focusing (Yang et al., 2016) and hologram (Li et al., 2017). In recent years, Cui et al. proposed the concept of reprogrammable coding metasurfaces, which can be dynamically controlled in microwave regimes (Cui et al., 2014; Li et al., 2017). Controlling the bias voltage applied across the PIN diode loaded on the top of the meta-atom can yield two different reflection phases, i.e., 0 and $\pi$ to mimic " 0 " and " 1 " states, respectively. Then, the phase distribution encoded on the whole metadevice can be controlled by directly reprogramming the bias voltages applied on these meta-atoms using field-programmable gate arrays (FPGA) directly. Therefore, real-time controllable digital beam steering (Cui et al., 2014; Wan et al., 2016) and dynamically switched holographic images can be realized (Li et al., 2017) (Figure 1C).

Although these digital reconfigurable metasurfaces can realize various functions, their beam steering ability is greatly limited because of 1-bit digital coding. Compared with them, the beam manipulation capability of 2-bit digital metasurface with two PIN diodes loaded on each meta-atom is obviously enhanced (Huang et al., 2017a). In 2017, Huang et al. proposed a 2-bit digitally controlled coding metasurface, which can dynamically switch between different scattering modes through a programmable electric source, such as beam deflection, multi beam and beam diffusion (Huang et al., 2017a). Moreover, a few recent works show the possibility to load switchable PIN diodes on Pancharatnam-Berry(PB) metasurface (Xu et al., 2016a) and water-based metasurface (Chen et al., 2019a). Xu et al. designed a tunable $\mathrm{PB}$ metasurface with frequency reconfigurability. By controlling the external voltages applied to the diodes, the operation band with $180^{\circ}$ phase difference between orthogonal reflection coefficients can be dynamically controlled. Therefore, when PIN diodes are "ON" state, the PB metasurface composed of these meta-atoms with orderly rotation angle exhibits a broadband photonic spin hall effect with nearly $100 \%$ conversion efficiency, when PIN diodes are "OFF" state, it switches to dual well-separated bands (Xu et al., 2016a). As for reconfigurable water-based metasurface integrated with PIN diodes, the EM wave reflected by the metasurface can be modulated by both the degree of salinity and the diode pattern. Through these two manipulating methods, the metasurface is able to control the amplitude of the scattered beams and the beam deflection angles, which provides a more flexible and economical way for wavefront manipulation (Chen et al., 2019a) (Figure 1D). With the deepening of the research, the manipulation of the transmitted electromagnetic wave by the reconfigurable metasurface is realized. Bai et al. (2020) proposed a transmissive reconfigurable metasurface for the generation of multi-mode convergent OAM beams with high efficiency (Figure 1E). Liu et al. (2020) demonstrated a dual-band realtime reconfigurable meta-atom with polarization-independent manipulation of reflection and transmission wavefronts.

\section{METASURFACES WITH VARACTORS}

\subsection{Uniformly Tuning With Varactors}

Thanks to its variable capacitance with more flexibility than PIN diode, varactor diode, a different electrically sensitive element, is an alternative excellent candidate for reconfigurable metasurfaces in microwave regime. The meta-atom could integrate a varactor diode to manipulate the electromagnetic response, where the capacitance of the varactor diode can be adjusted in a continuous way. In the simplest case, all varactors are controlled by the same voltage, which effectively provides frequency tunability for the metasurface (Mias and Yap, 2007; Zhu et al., 2013; Dincer, 2015). The most widely investigated functionality is tunable perfect absorption.By adjusting the reverse bias voltage loaded on the varactor diode, the absorption frequency of the designed unit can be controlled continuously (Zhao et al., 2013; Lin et al., 2014; Zhu et al., 2015). For instance, in 2016, Luo and coworkers (Luo et al., 2016) experimentally demonstrated an electrically tunable metasurface absorber in the $\mathrm{GHz}$ regime based on dissipating behavior of embedded varactors (Figure 2A). Due to the varactors and biasing circuits embedded in the unit structure, the absorptivity can be tuned with a wide range by changing DC biasing voltage. In addition, employing varactors on metasurfaces enables tunable frequency and phase properties (Zhu et al., 2013). 

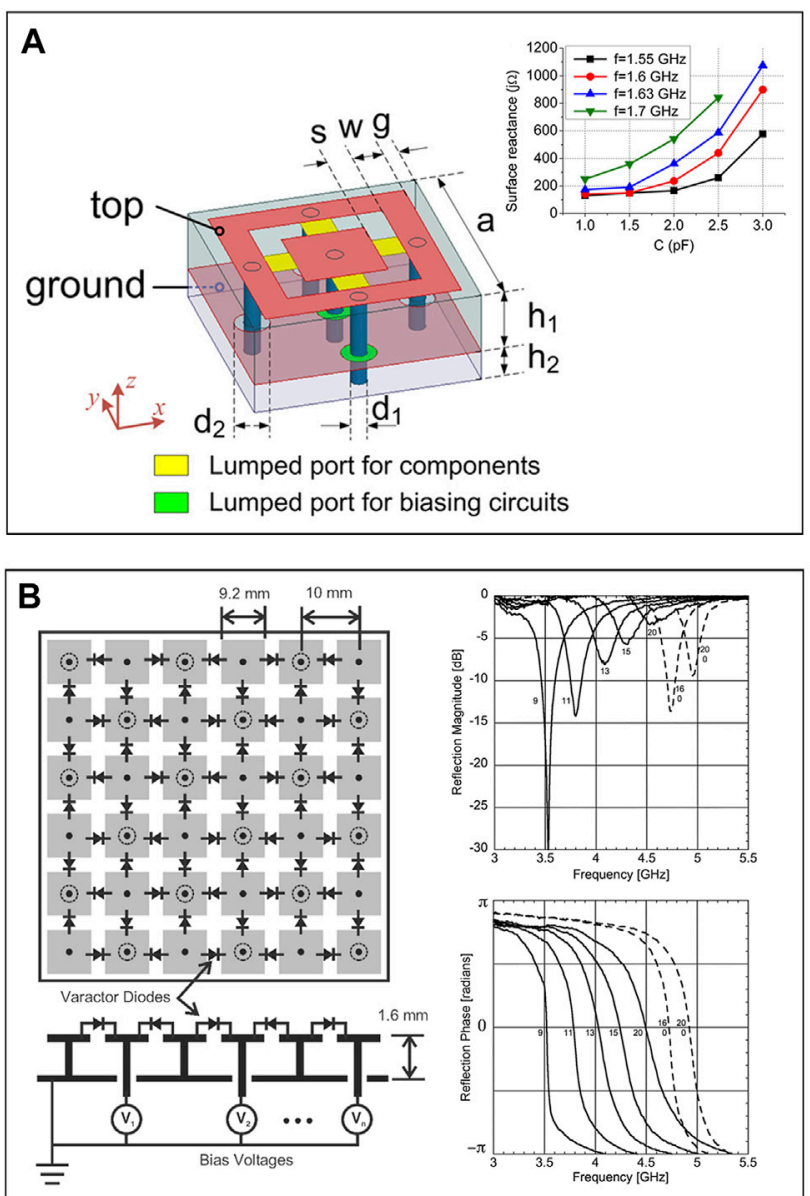
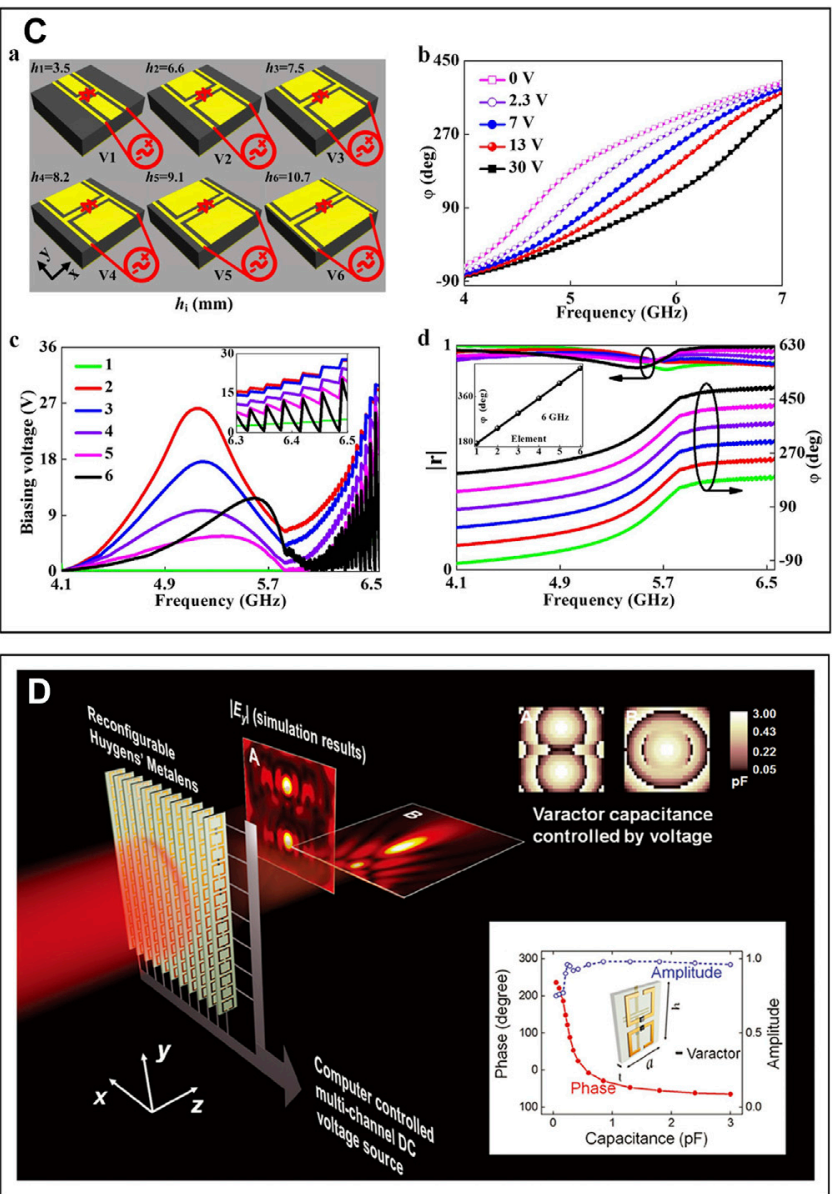

FIGURE 2 | (A) Electrically tunable metasurface absorber with embedded varactors. Reproduced from Luo et al. (2016) with permission from AIP Publishing. (B) Active metasurface with full $360^{\circ}$ reflection phase tuning. Reproduced from Zhu et al. (2013) with permission from Spring Nature. (C) Tunable microwave metasurfaces with dynamical switch. Reproduced from Xu et al. (2016b) with permission from Spring Nature. (D) Reconfigurable Huygens' metalens. Reproduced from Chen et al. (2017) with permission from John Wiley and Sons.

In this case, the meta-atom, a multiple resonance structure with two resonance poles and one resonance zero, is capable of providing full $2 \pi$ reflection phase variation, active tuning in finite frequency and linear reflection phase tuning (Figure 2B). Zhu et al. (2010) demonstrated a tunable metamaterial reflector/absorber for polarization modulation, which is made of ELC resonators with varactor diodes. The EM reflections for orthogonal polarized incident waves can be tuned independently by adjusting the bias voltages on the corresponding diodes. Because of this characteristic, the reflected waves can be electrically controlled to a linear polarization with polarizaiton azimuth angle tunable from $0^{\circ}$ to $90^{\circ}$ at the resonant frequency. When off the resonant frequency, an elliptical polarization with tunable azimuth angle of the major axis can be generated.

\subsection{Non-Uniformly Tuning With Varactors}

The locally applied tuning voltages could be different for each unit cell of the metasurface with non-uniformly tuning varactors. Nearly 20 years ago, Sievenpiper and coworkers (Sievenpiper et al., 2003) proposed an electrically steerable reflector based on a resonant textured surface loaded with varactor diodes in the microwave regime. Researchers demonstrated that by programming the reflective phase gradient on the meta-device, the reflected beam can be electrically controlled over $\pm 40^{\circ}$ for both polarizations. Inspired by this work, many other microwave metasurfaces were proposed to perform different functionalities, such as tunable frequency and phase properties (Burokur et al., 2010; Dai et al., 2018), beam scanning (Ratni et al., 2018a), beam focusing (Xu et al., 2016c; Lv et al., 2019; Wang et al., 2019), hologram (Zhang K. et al., 2018), pre-designed scattering field generation (Huang et al., 2018; Li T. et al., 2019) and vortex beam generation (Guo et al., 2020). Xu et al. (2016b) established a tunable scheme to overcome the dispersion-induced issues in microwave metasurfaces. By adding tunable elements to our meta-atoms, the phase response of each meta-atom can be precisely controlled via external knobs, so as to correct the inevitable phase distortion at any frequency (Figure 2C). Ratni et al. (2018a) experimentally demonstrated an active metasurface 
for reconfigurable reflectors, which can produce anomalous reflection properties within a broad frequency range and scan the direction of the reflected beam in a certain angle range. In addition, this kind of reconfigurable planar metasurface is of frequency agility and can realize beam focusing (Ratni et al., 2018b).

The above active meta-devices work in reflection mode. Reflection mode is relatively easy to implement, but sometimes it is not conducive to some applications. In order to achieve tunable/reconfigurable meta-devices in transmission mode, it is necessary to precisely control the phase and amplitude of the locally transmitted wave through each meta-atom. So far, reconfigurable pixel metasurfaces in transmission mode are already available in some areas, such as vortex beam generation (Shi et al., 2019), dynamical focusing (Chen et al., 2017) and so on. In 2017, Chen et al. (2017) experimentally presented a tunable microwave Huygens' metasurface with dynamically controllable focal point (Figure 2D). Shi et al. proposed an active metasurface for multiple vortex beams $(l=$ $+1,0,-1$ ) generation (Shi et al., 2019). Different modes vortex beams generation can be realized by changing the transmission phase distribution on the metasurface aperture. Beyond that, Masud et al. proposed a dual-band compact tunable metasurface for EM interference shielding (Masud et al., 2012). By loading varactor diodes on the metasurface, the lower shielding band can be tuned without affecting the resonant frequency of the upper shielding band. The measurement results show that the center frequency of the lower shielding band can be tuned by $80 \mathrm{MHz}$.

In recent years, the research of tunable devices with inverse design by deep learning has become a hot spot. Yoya et al. (2019) proposed a self-adaptive invisibility metasurface cloak driven by deep learning. At microwave frequency, the reflection characteristics of each meta-atom inside the tunable metasurface can be changed independently by applying different DC bias on the loaded varactor diode. By introducing an embedded pre-trained artificial neural network, the metasurface cloak is capable of responding rapidly to the fastchanging incident wave and surrounding background.

\section{DISCUSSION}

In this mini review, we have briefly summarized the recent progress on electrically reconfigurable microwave metasurfaces tuned uniformly and non-uniformly by different types of active lumped elements. The tuning mechanism and potential applications of the reconfigurable microwave metasurface were introduced. It is worth noting that a more comprehensive way for designing electrically reconfigurable metasurfaces is to combine multiple tunable elements (e.g. PIN diode and varactor), which

\section{REFERENCES}

Akram, M. R., Ding, G., Chen, K., Feng, Y., and Zhu, W. (2020). Ultrathin Single Layer Metasurfaces With Ultra-Wideband Operation for Both provides the possibility to achieve multiple EM functionalities and real-time reconfigurability in one design simultaneously ( $\mathrm{Wu}$ et al., 2013; Huang et al., 2017b). For example, Wu et al. (2013) presented and experimentally characterized a microwave active absorber which has dual-ability of simultaneous but dividable modulation on absorbing frequency and intensity. Huang et al. (2017b) reported a reconfigurable metasurface for multifunctional control of EM waves. By controlling tunable elements, the proposed metasurface can dynamically change its local phase distribution to generate pre-designed EM responses. Recently, there are many researches on the application of using tunable devices in antenna area (Yoya et al., 2019; Esmaeili and Laurin, 2020). For instance, in 2020, Esmaeili and Laurin (2020) proposed a cylindrical dielectric resonator antenna, which are able to switch between linear horizontal, linear vertical and circular polarizations. Futhermore, active amplifiers provide another option for continuously tunable pixel reconfigurable metasurfaces (Chen et al., 2019b). A spatial-energy digital-coding metasurface with active amplifiers is proposed to realize arbitrary editing of the energy of spatial propagating waves in the microwave frequency range. Meanwhile, the concept of space-time metasurface, which characterized by spatially and temporally variant properties, has been proposed (Zhang L. et al., 2018; Zhang C. et al., 2020; Castaldi et al., 2021). It is meaningful to exploit the temporal dimension by applying a dynamic switching of the coding sequence. Most recently, graphene, a famous electrically sensitive material, has been utilized for designing electrically reconfigurable metasurfaces at microwave frequencies (Zhang et al., 2019; Zhang et al., 2021). However, due to the limited scope of this mini review, we do not summary such types of reconfigurable metasurfaces in this mini review.

The rapid development of reconfigurable metasurfaces from both global and local tuning significantly expands the ability of electromagnetic wave manipulations. The electrically reconfigurable metasurfaces have the potential to automatically adapt to environmental changes. We believe new ideas and new designs will surely emerge in the near future with various new applications, in addition to those described in this review.

\section{AUTHOR CONTRIBUTIONS}

LS wrote the first draft and WZ reviewed/edited this paper.

\section{FUNDING}

This work was supported by the National Natural Science Foundation of China (NSFC) (62071291 and 51777168).

Transmission and Reflection. Adv. Mater. 32, 1907308. doi:10.1002/ adma.201907308

Akram, M. R., Mehmood, M. Q., Bai, X. D., Jin, R. H., Premaratne, M., and Zhu, W. R. (2019). High Efficiency Ultrathin Transmissive Metasurfaces. Adv. Opt. Mater. 7, 1801628. doi:10.1002/adom.201801628 
Bai, X. D., Kong, F. W., Sun, Y. T., Wang, G. F., Qian, J. Y., Li, X. B., et al. (2020). High-Efficiency Transmissive Programmable Metasurface for Multimode Oam Generation. Adv. Opt. Mater. 8, 2000570. doi:10.1002/ adom.202000570

Burokur, S. N., Daniel, J. P., Ratajczak, P., and de Lustrac, A. (2010). Tunable Bilayered Metasurface for Frequency Reconfigurable Directive Emissions. Appl. Phys. Lett. 97, 509. doi:10.1063/1.3478214

Castaldi, G., Zhang, L., Moccia, M., Hathaway, A. Y., Tang, W. X., Cui, T. J., et al. (2021). Joint Multi-Frequency Beam Shaping and Steering via Space-TimeCoding Digital Metasurfaces. Adv. Funct. Mater. 31, 2007620. doi:10.1002/ adfm. 202007620

Chen, H. T., Taylor, A. J., and Yu, N. (2016). A Review of Metasurfaces: Physics and Applications. Rep. Prog. Phys. 79, 076401. doi:10.1088/0034-4885/79/7/076401

Chen, K., Feng, Y., Monticone, F., Zhao, J., Zhu, B., Jiang, T., et al. (2017). A Reconfigurable Active Huygens' Metalens. Adv. Mater. 29, 1606422. doi:10. 1002/adma.201606422

Chen, L., Ma, H. L., Ruan, Y., and Cui, H. Y. (2019a). Dual-Manipulation on WaveFront Based on Reconfigurable Water-Based Metasurface Integrated With Pin Diodes. J. Appl. Phys. 125, 023107. doi:10.1063/1.5078660

Chen, L., Ma, Q., Jing, H. B., Cui, H. Y., Liu, Y., and Cui, T. J. (2019b). Space-Energy Digital-Coding Metasurface Based on an Active Amplifier. Phys. Rev. Appl. 11, 125. doi:10.1103/PhysRevApplied.11.054051

Cui, T. J., Qi, M. Q., Wan, X., Zhao, J., and Cheng, Q. (2014). Coding Metamaterials, Digital Metamaterials and Programmable Metamaterials. Light Sci. Appl. 3, e218. doi:10.1038/lsa.2014.99

Dai, J. Y., Zhao, J., Cheng, Q., and Cui, T. J. (2018). Independent Control of Harmonic Amplitudes and Phases via a Time-Domain Digital Coding Metasurface. Light Sci. Appl. 7, 90. doi:10.1038/s41377-018-0092-z

Dincer, F. (2015). Electromagnetic Energy Harvesting Application Based on Tunable Perfect Metamaterial Absorber. J. Electromagn. Waves Appl. 29, 2444-2453. doi:10.1080/09205071.2015.1027794

Esmaeili, M., and Laurin, J. J. (2020). Polarization Reconfigurable Slot-Fed Cylindrical Dielectric Resonator Antenna. Prog. Electromagn. Res. 168, 61-71. doi:10.2528/PIER20041203

Ghosh, S., and Srivastava, K. V. (2016). Polarisation-Independent Switchable Absorber/Reflector. Electron. Lett. 52, 1141-1143. doi:10.1049/el.2016.1193

Guo, K., Zheng, Q., Yin, Z. P., and Guo, Z. Y. (2020). Generation of ModeReconfigurable and Frequency-Adjustable Oam Beams Using Dynamic Reflective Metasurface. IEEE Access 8, 75523-75529. doi:10.1109/access. 2020.2988914

Huang, C., Sun, B., Pan, W., Cui, J., Wu, X., and Luo, X. (2017a). Dynamical Beam Manipulation Based on 2-Bit Digitally-Controlled Coding Metasurface. Sci. Rep. 7, 42302. doi:10.1038/srep42302

Huang, C., Yang, J. N., Wu, X. Y., Song, J. K., Pu, M. B., Wang, C. T., et al. (2018). Reconfigurable Metasurface Cloak for Dynamical Electromagnetic Illusions. ACS Photon. 5, 1718. doi:10.1021/acsphotonics.7b01114

Huang, C., Zhang, C. L., Yang, J. N., Sun, B., Zhao, B., and Luo, X. G. (2017b). Reconfigurable Metasurface for Multifunctional Control of Electromagnetic Waves. Adv. Opt. Mater. 5, 1700485. doi:10.1002/adom.201700485

Li, L. L., Cui, T. J., Ji, W., Liu, S., Ding, J., Wan, X., et al. (2017). Electromagnetic Reprogrammable Coding-Metasurface Holograms. Nat. Commun. 8, 197. doi:10.1038/s41467-017-00164-9

Li, T., Yang, H. H., Li, Q., Zhang, C., Han, J. F., Cong, L. L., et al. (2019). Active Metasurface for Broadband Radiation and Integrated Low Radar Cross Section. Opt. Mater. Express 9, 1161-1172. doi:10.1364/Ome.9.001161

Li, W., Xia, S., He, B., Chen, J., Shi, H., Zhang, A., et al. (2016). A Reconfigurable Polarization Converter Using Active Metasurface and its Application in Horn Antenna. IEEE Trans. Antennas Propag. 64, 5281-5290. doi:10.1109/TAP.2016. 2620484

Li, Y., Lin, J., Guo, H., Sun, W., Xiao, S., and Zhou, L. (2020). A Tunable Metasurface With Switchable Functionalities: From Perfect Transparency to Perfect Absorption. Adv. Opt. Mater. 8, 1901548. doi:10.1002/adom.201901548

Li, Y., Wang, Y., and Cao, Q. S. (2019). Design of a Multifunctional Reconfigurable Metasurface for Polarization and Propagation Manipulation. IEEE Access 7, 129183-129191. doi:10.1109/Access.2019.2939200

Li, Z., Premaratne, M., and Zhu, W. (2020). Advanced Encryption Method Realized by Secret Shared Phase Encoding Scheme Using a Multi-Wavelength Metasurface. Nanophotonics 9, 3687-3696. doi:10.1515/nanoph-2020-0298
Lin, B. Q., Da, X. Y., Zhao, S. H., Meng, W., Li, F., Fang, Y. W., et al. (2014). Design of a Varactor-Tunable Metamaterial Absorber. Chin. Phys. B 23, 542-546. doi:10.1088/1674-1056/23/6/067801

Liu, G. Y., Liu, H. X., Han, J. Q., Mu, Y. J., and Li, L. (2020). Reconfigurable Metasurface with Polarization-Independent Manipulation for Reflection and Transmission Wavefronts. J. Phys. D 53, 045107. doi:10.1088/1361-6463/ ab5253

Liu, Y., and Zhang, X. (2011). Metamaterials: A New Frontier of Science and Technology. Chem. Soc. Rev. 40, 2494-2507. doi:10.1039/c0cs00184h

Luo, Z. J., Long, J., Chen, X., and Sievenpiper, D. (2016). Electrically Tunable Metasurface Absorber Based on Dissipating Behavior of Embedded Varactors. Appl. Phys. Lett. 109, 1516-1519. doi:10.1063/1.4961367

Lv, Y. H., Ding, X., Wang, B. Z., and Wang, R. (2019). Wideband PolarisationInsensitive Metasurface With Tunable Near-Field Scattering Focusing Characteristic. Electron. Lett. 55, 776-777. doi:10.1049/el.2019.1275

Masud, M. M., Ijaz, B., Ullah, I., and Braaten, B. (2012). A Compact Dual-Band Emi Metasurface Shield With an Actively Tunable Polarized Lower Band. IEEE Trans. Electromag. Compat. 54, 1182-1185. doi:10.1109/Temc.2012.2210899

Mias, C., and Yap, J. H. (2007). A Varactor-Tunable High Impedance Surface With a Resistive-Lumped-Element Biasing Grid. IEEE Trans. Antennas Propag. 55, 1955-1962. doi:10.1109/Tap.2007.900228

Qi, Z., Zhongxiang, S., Jianpeng, W., and Kian Seng, L. (2012). Design of a Switchable Microwave Absorber. IEEE Antennas Wirel. Propag. Lett. 11, 1158-1161. doi:10.1109/lawp.2012.2220115

Ratni, B., de Lustrac, A., Piau, G. P., and Burokur, S. N. (2018a). Active Metasurface for Reconfigurable Reflectors. Appl. Phys. A. 124, 104. doi:10.1007/s00339-017$1502-4$

Ratni, B., de Lustrac, A., Piau, G. P., and Burokur, S. N. (2018b). Reconfigurable Meta-Mirror for Wavefronts Control: Applications to Microwave Antennas. Opt. Express 26, 2613-2624. doi:10.1364/OE.26.002613

Shao, L. D., Zhu, W. R., Leonov, M. Y., and Rukhlenko, I. D. (2019). Dielectric 2-Bit Coding Metasurface for Electromagnetic Wave Manipulation. J. Appl. Phys. 125, 203101. doi:10.1063/1.5094561

Shi, H., Wang, L., Peng, G., Chen, X., Li, J., Zhu, S., et al. (2019). Generation of Multiple Modes Microwave Vortex Beams Using Active Metasurface. IEEE Antennas Wirel. Propag. Lett. 18, 59-63. doi:10.1109/lawp.2018.2880732

Sievenpiper, D. F., Schaffner, J. H., Song, H. J., Loo, R. Y., and Tangonan, G. (2003). Two-Dimensional Beam Steering Using an Electrically Tunable Impedance Surface. IEEE Trans. Antennas Propag. 51, 2713-2722. doi:10.1109/Tap.2003.817558

Sun, S., He, Q., Hao, J., Xiao, S., and Zhou, L. (2019). Electromagnetic Metasurfaces: Physics and Applications. Adv. Opt. Photon. 11, 380-479. doi:10.1364/AOP.11. 000380

Sun, S., He, Q., Xiao, S., Xu, Q., Li, X., and Zhou, L. (2012). Gradient-Index MetaSurfaces as a Bridge Linking Propagating Waves and Surface Waves. Nat. Mater. 11, 426-431. doi:10.1038/nmat3292

Tao, Z., Wan, X., Pan, B. C., and Cui, T. J. (2017). Reconfigurable Conversions of Reflection, Transmission, and Polarization States Using Active Metasurface. Appl. Phys. Lett. 110, 121901. doi:10.1063/1.4979033

Wan, X., Qi, M. Q., Chen, T. Y., and Cui, T. J. (2016). Field-Programmable Beam Reconfiguring Based on Digitally-Controlled Coding Metasurface. Sci. Rep. 6, 20663. doi:10.1038/srep20663

Wang, Z. H., Liao, D. S., Zhang, T., Chen, T. H., Ruan, Y., and Zheng, B. (2019). Metasurface-Based Focus-Tunable Mirror. Opt. Express 27, 30332-30339. doi:10.1364/OE.27.030332

Wu, X., Hu, C., Wang, Y., Pu, M., Huang, C., Wang, C., et al. (2013). Active Microwave Absorber With the Dual-Ability of Dividable Modulation in Absorbing Intensity and Frequency. AIP Adv. 3, 022114. doi:10.1063/1. 4792069

Xu, H. X., Ma, S., Luo, W., Cai, T., Sun, S., He, Q., et al. (2016c). Aberration-Free and Functionality-Switchable Meta-Lenses Based on Tunable Metasurfaces. Appl. Phys. Lett. 109, 333. doi:10.1063/1.4967438

Xu, H. X., Tang, S. W., Ma, S. J., Luo, W. J., Cai, T., Sun, S. L., et al. (2016b). Tunable Microwave Metasurfaces for High-Performance Operations: Dispersion Compensation and Dynamical Switch. Sci. Rep. 6, 38255. doi:10.1038/ srep38255

Xu, H. X., Wang, G. M., Cai, T., Xiao, J., and Zhuang, Y. Q. (2016a). Tunable Pancharatnam-berry Metasurface for Dynamical and High-Efficiency Anomalous Reflection. Opt. Express 24, 27836-27848. doi:10.1364/Oe.24.027836 
Xu, X. X., Jiang, J. J., Miao, L., Chen, Q., and Sun, B. A. (2012). Design of Tunable Metamaterial Absorbers Based on Pin Diodes. IEICE Electron. Express 9, 1408-1413. doi:10.1587/elex.9.1408

Yang, H. H., Cao, X. Y., Yang, F., Gao, J., Xu, S. H., Li, M. K., et al. (2016). A Programmable Metasurface with Dynamic Polarization, Scattering and Focusing Control. Sci. Rep. 6, 35692. doi:10.1038/srep35692

Yoo, M., and Lim, S. (2014). Active Metasurface for Controlling Reflection and Absorption Properties. Appl. Phys. Express 7, 112204. doi:10.7567/apex.7. 112204

Yoya, A. C. T., Fuchs, B., and Davy, M. (2019). A Reconfigurable Chaotic Cavity With Fluorescent Lamps for Microwave Computational Imaging. Prog. Electromagn. Res. 165, 1-12. doi:10.2528/PIER19011602

Yu, N., Genevet, P., Kats, M. A., Aieta, F., Tetienne, J.-P., Capasso, F., et al. (2011). Light Propagation With Phase Discontinuities: Generalized Laws of Reflection and Refraction. Science 334, 333-337. doi:10.1126/science. 1210713

Zhang, C., Yang, J., Yang, L. X., Ke, J. C., Chen, M. Z., Cao, W. K., et al. (2020). Convolution Operations on Time-Domain Digital Coding Metasurface for Beam Manipulations of Harmonics. Nanophotonics 9, 2771-2781. doi:10.1515/ nanoph-2019-0538

Zhang, J., Li, Z., Shao, L., and Zhu, W. (2021). Dynamical Absorption Manipulation in a Graphene-Based Optically Transparent and Flexible Metasurface. Carbon 176, 374-382. doi:10.1016/j.carbon.2021.01.137

Zhang, J., Wei, X., Rukhlenko, I. D., Chen, H. T., and Zhu, W. (2020a). Electrically Tunable Metasurface With Independent Frequency and Amplitude Modulations. ACS Photon. 7, 265-271. doi:10.1021/acsphotonics.9b01532

Zhang, J., Wei, X. Z., Premaratne, M., and Zhu, W. R. (2019). Experimental Demonstration of an Electrically Tunable Broadband Coherent Perfect Absorber Based on a Graphene-Electrolyte-Graphene Sandwich Structure. Photon. Res. 7, 868-874. doi:10.1364/Prj.7.000868

Zhang, J., Zhang, H., Yang, W. X., Chen, K., Wei, X. Z., Feng, Y. J., et al. (2020b). Dynamic Scattering Steering With Graphene-Based Coding Metamirror. Adv. Opt. Mater. 8, 2000683. doi:10.1002/adom.202000683
Zhang, K., Yu, H., Ding, X., and Wu, Q. (2018). Experimental Validation of Active Holographic Metasurface for Electrically Beam Steering. Opt. Express 26, 6316-6324. doi:10.1364/OE.26.006316

Zhang, L., Chen, X. Q., Liu, S., Zhang, Q., Zhao, J., Dai, J. Y., et al. (2018). SpaceTime-Coding Digital Metasurfaces. Nat. Commun. 9, 4334. doi:10.1038/ s41467-018-06802-0

Zhao, J., Cheng, Q., Chen, J., Qi, M. Q., Jiang, W. X., and Cui, T. J. (2013). A Tunable Metamaterial Absorber Using Varactor Diodes. New J. Phys. 15, 043049. doi:10.1088/1367-2630/15/4/043049

Zhao, R., Gong, B., Xiao, F., He, C., and Zhu, W. (2019). Circuit Model Analysis of Switchable Perfect Absorption/reflection in an Active Frequency Selective Surface. IEEE Access 7, 55518-55523. doi:10.1109/access.2019.2913406

Zheludev, N. I., and Kivshar, Y. S. (2012). From Metamaterials to Metadevices. Nat. Mater. 11, 917-924. doi:10.1038/nmat3431

Zhu, B., Feng, Y., Zhao, J., Huang, C., Wang, Z., and Jiang, T. (2010). Polarization Modulation by Tunable Electromagnetic Metamaterial Reflector/absorber. Opt. Express 18, 23196-23203. doi:10.1364/OE.18.023196

Zhu, B. O., Zhao, J. M., and Feng, Y. J. (2013). Active Impedance Metasurface With Full 360 Degrees Reflection Phase Tuning. Sci. Rep. 3, 3059. doi:10.1038/srep03059

Zhu, J. F., Li, D. L., Yan, S., Cai, Y. J., Liu, Q. H., and Lin, T. (2015). Tunable Microwave Metamaterial Absorbers Using Varactor-Loaded Split Loops. EPL 112, 54002. doi:10.1209/0295-5075/112/54002

Conflict of Interest: The authors declare that the research was conducted in the absence of any commercial or financial relationships that could be construed as a potential conflict of interest.

Copyright (c) 2021 Shao and Zhu. This is an open-access article distributed under the terms of the Creative Commons Attribution License (CC BY). The use, distribution or reproduction in other forums is permitted, provided the original author(s) and the copyright owner(s) are credited and that the original publication in this journal is cited, in accordance with accepted academic practice. No use, distribution or reproduction is permitted which does not comply with these terms. 